Correction

\title{
Correction: Rubin, J.L. Applications of a Particular Four-Dimensional Projective Geometry to Galactic Dynamics. Galaxies 2018, 6, 83
}

\author{
Jacques L. Rubin $(1)$ \\ Institut de Physique de Nice-UMR7010-UNS-CNRS, Université de Nice Sophia Antipolis, Site Sophia Antipolis, \\ 1361 route des lucioles, 06560 Valbonne, France; jacques.rubin@inphyni.cnrs.fr; Tel.: +33-(0)4-92-96-7323 \\ Received: 9 October 2018; Accepted: 9 October 2018; Published: 19 October 2018 \\ check for \\ updates
}

The author wishes to make the following corrections to this paper [1]:

The abstract begins with the following sentence: "Relativistic location systems that extend relativistic positioning systems ... geometry." The word "location" in this sentence is inappropriate and should be substituted by the word "localizing".

In Page 2, the second paragraph starts with the sentence: "This projective geometry that relativistic localization systems unveil ... geometry." Again, this sentence should be corrected as follows: "This projective geometry that relativistic localizing systems unveil ... geometry.", where the word "localization" is substituted by "localizing".

The expression "relativistic localizing systems" is generic, unlike the incorrect expressions "relativistic location/localization systems", which, consequently, should not be used; hence, these corrections.

The author apologizes for any inconvenience this change might cause. This correction does not cause any changes to the results and the conclusions in the original published paper. The manuscript will be updated and the original will remain online on the article webpage.

\section{Reference}

1. Rubin, J.L. Applications of a Particular Four-Dimensional Projective Geometry to Galactic Dynamics. Galaxies 2018, 6, 83. [CrossRef]

2018 by the author. Licensee MDPI, Basel, Switzerland. This article is an open access article distributed under the terms and conditions of the Creative Commons Attribution (CC BY) license (http://creativecommons.org/licenses/by/4.0/). 